50 prozentiger Lösung von Natriumvalerianat mit $10 \mathrm{ccm}$ Kresol 17 ccm Benzol klar auf.

Kampher befördert in gleicher Weise die Löslichkeit der Kohlenwasserstoffe in Seifen.

Die Kresole lösen sich anch in zahlreichen anderen wässrigen Salzlösungen mehr oder weniger auf, so - abgesehen von den Kresotinaten, Kresolaten etc., dem Solveole, Solitole u. a. - in den Salzen des Ammoniaks und Anilins mit Salicylsäure, Benzoësäure. Phenylessigsäure u. a. m., durchweg jedvch in geringerer Menge als in Seifenlösungen.

Endlich machen wir bei dieser Gelegenheit auch auf das Glycerin als ein ausgezeichnetes Lösungsmittel für Kresole aufmerksam; $9 \mathrm{cmm}$ Glycerin lösen $1 \mathrm{~g} \mathrm{p}$-Kresol klar auf, and die Lösung kann mit ihrem gleichen Volum Wasser verdünnt werden, bis Trübung eintritt; das o-Kresol ist noch löslicher, $1 \mathrm{~g}$ desselben gebraucht nur $4 \mathrm{ccm}$ Glycerin zur vollständigen Lösung. Die Lösung eines Gemisches von $1 \mathrm{~g} \mathrm{o}$-Kresol und $1 \mathrm{~g} \mathrm{p}$-Kresol in $13 \mathrm{ccm}$ Glycerin ist in allen Verhältnissen mit Wasser klar mischbar.

\title{
Mitteilungen aus dem chem.-pharm. Laboratorium der Herzogl. techn. Hochschule zu Braunschweig. Beiträge zur chemischen und pharmakognosti- schen Kenntnis der Kakaobohnen \\ von
}

H. Beckurts and C. Hartwich.

(Eingegangen den 15. VII. 1892.)

Bekanntlich hat $P$. Trojanowsky ${ }^{1}$ ) aus den abweichenden chemischen Reaktionen, welche er auf Zusatz verschiedener Reagentien zu den wässerigen Auszügen der Kakaobohnen verschiedener Provenienz erhielt, einen analytischen Gang abgeleitet, um diese von einander $\mathrm{zu}$ unterscheiden.

P. Zipperer ${ }^{2}$ ) hat sodann in seiner wertvollen Arbeit über die Untersuchung von Kakao und dessen Präparaten eine Methode

1) Arch. Pharm. 1877, 210, 30.

2) Untersuchungen über Kakao und dessen Präparatc. Hambu:g und Teipzig. I. Vols. $188 \%$. 
zur Unterscheidung der verschiedenen Handelssorten der Kakaobohnen vorgeschlagen, welche sich anf die Beschaffenheit der im Embryo enthaltenen Pigmentzellen gründet. Im Anschlufs daran wurde sodann von demselben Autor die Frage geprüft, ob das Pigment der Kakaobohnen eines jeden Jahrgangs in derselben Handelssorte das Gleicbe ist, oder Unterschiede existieren. War letzteres der Fall, so mulsten dieselben Reagentien, welche Trojanowsky 1874 angewendet hatte, heute andere Ergebnisse liefern; war ersteres richtig, so mulsten die Reaktionen die Gleichen bleiben. Zipperer hat nun bei der Wiederholung der Versuche Trojanowsky's dieselben Reaktionen erhalten und daraus geschlossen, dafs die verschiedenen Jahrgänge der gleichen Kakaosorten, wass das Pigment anbelangt, nicht unter einander verschieden sind und sich auch Kakaobohnen gleicher Abstammung unter einander in der Färbung sowohl, wie in den Reaktionen, welche sio bei Anwendung geeigneter Reagentien zeigen, gleichen.

Der eine von uns hat schon vor einiger Zeit ${ }^{3}$ ) die Angaben Zipperer's, soweit dieselben sich auf die Beschaffenheit der Pigmentzellen und das in ihnen enthaltene Pigment beziehen, einer Nachprüfung unterzogen und ist dabei zu Resultaten gelangt, die von denen Zipperer's durchaus abweichen, so dafs damals die Ansicht ausgesprochen wurde, dass kein Mikroskopiker auf Grund der Untersuchung der Pigmentzellen eine Handelssorte erkennen könnte.

Eine gröfsere Kollektion Kakaobohnen verschiedener Provenienz, welche uns von der Firma Wittekop \& Co. in Braunschweig zur Verfügung gestellt wurde, wofür wir derselben auch an dieser Stelle unseren verbindlichsten Dank aussprechen, gab Veranlassung, die Untersuchungen Trojanowsky's und Zipperer's wieder aufzunehmen, um die noch nicht genügend geklärte Frage der Unterscheidung der einzelnen Handelssorten möglichst zu entscheiden. Auch bei diesen neuen Untersuchnngen hat sich sowohl hinsichtlich der Beschaffenheit der Pigmentzellen, wie auch der Reaktionen bei Anwendung geeigneter Reagentien keine hinreichende Übereinstimmung mit den Resultaten Zipperer's und Trojanowsky's ergeben, aber

3) Arch. Pharm. 1887, 554. 
auch die C̈hereinstimmnng mit den Resultaten der Untersuchung von 185 ist eine so wenigr befriedigende. dafs die damals ausgesprochene und soeben angeffihte Insicht aufrecht erhalten l,leiben mul's. Von 8 Sorten, die 1887 (von Peikert \& Co. in Wernigerode bezogen! untersucht wurden und denen jetzt eine gleiche Anzahl ebenso hezeichneter gregenüber gestellt werden konnten. stimmen 3 Sorten (Porto ('abello. Nachala und Bahia) uberein, 3 Sorteu (Caracas. St. Thomas und Trinidad) stimmen nicht und bei 2 Sorten (Ceylon und Ariha) ist die (Tbereinstimmung nur eine teilweise.

Es knun nicht. Wunder nelmnen. dall's die Hundelssorten bezüglich der Farhe, des Querschnittes und les Pigmenties wenig konstant sind. Wir wissen. dal's das Pigment, aus einem (rerbstoff entsteht und dem Anthocvan mindestens sehr nahe verwandt ist, und es ist leicht moglich. hal's diese [imwandlung von zahlreichen Falstoren (z. B. Reitezustand) abhängi,r ist. die wir nirht kennen, in, dafs sie nicht solten gan\% unterbleibt: jedentalls geht aus der nachlfolgenden Übersicht hervor. lafs es Handelssorten giebt, welche gar kein Pignent, sonclern nur ('erbstoff in besonderen Zellen enthalten und dals es zweiten: Sorten giebt, die eine geringe Menge Farbstoff durch das Parenchym mehr oder weniger verteilt onthalten, was allordings zuweilen erst hei der Behandlung mit Alkalion an der auftretenden Grüntürbıngr erkannt werden kann.

Ferner wurde bereits $188 \overline{\text { T }}$ der Standpunkt vertreteu. dal's die ganz allgemein verbreitete Ansicht. dilis der violette Farbstoff durch den eigentümlichen Ciuhrungsprozelis, dus Rotten, in hraun umgrwandelt ist. nicht richtig ist. Diese Ansicht ist durch unsere nenen Untersuchungen nur hefestigt worden, da granz unzweifelhat't gerottete Samen violetten Farbstoff zeigen. Fs ist überhaupt nicht mehr möglich. eine gerottete Bohne von einer ungerotteten schon äufserlich dadurch zu unterscheiden, dafs die gerottete cinen therzug ron der Erde zeigt, in welche die Bolnen zun /awecke des Ruttens eingegraben wurden, da diese primitive Methode an mindestens zahlreichen Produktionsorten verlassen und durch rationellere Einrichtungen. Lei denen die Bohnen in hölzerne oder metallene Gefäfss: gelangon. also mit der Erde ja nicht mehr in Beruhrung komunen. ersetzt ist. Da aber oft uns alter Gewohnheit verlangt wird, dafis eine gute Bohne ein erdiges Ansehen habe, so wird ibnen ein ẗbcr- 
zug von meist rötlicher Erde zuweilen künstlich gegeben. Nach. einer Nachricht soll diesem Üherzug sogar Zinnober zugesetzt werden.

Nach unserer Ansicht ist eine gerottete von einer ungerotteten Bohne mit einiger Sicherheit nur durch den Geschmack zu unterscheiden, da ja die den ungerotteten Bohnen. eigentümliche Bitterkeit und Herbigkeit durch das Rotten verschwindet und der eigentümliche aromatische, ja zuweilen fast ätherartige Geschmack erst dadurch entwickelt wird. - Ferner ist dabei noch auf einen andern Punkt die Aufmerksamkeit zu lenken. Die Parenchymzellen der Samenlappen, die die weit überwiegende Hauptmasse des Gewebes ausmachen, enthalten neben Öl, Stärke u. s. w. in dem Zellinhalt eine geringe Menge Gerbstoff, der mit Eisenchlorid unschwer nachzuweisen ist. Bei zahlreichen Sorten nun hat dieser Gerbstoff eine Umwandlung erlitten, indem er in einen braunen Farbstoff, ein Phlobaphen, ganz oder teilweise übergegangen ist und zwar hat diese Umwandlung die peripheren Partien besonders betroffen. Unter dem Mikroskop erscheint die Menge dieses braunen Farbstoffes meist nur gering, ist aber für die ganzen Samen doch oft genug von wesentlicher Bedeutung, da er für die Färbung des Querschnitts sehr ins Gewicht tällt. Wie die nachtolgende Übersicht erkennen lärst, zeigen manche Sorten, trotzdem. sie violetten oder violettroten Farbstoff enthalten einen braunen Querschnitt, welche letztere Farbe dann auf Rechnung des in den Parenchymzellen enthaltenen Phlobaphens zu setzen ist. Es wäre nun nicht unmöglich, dafs die Bildung dieses Phlobaphens durch das Rotten begünstigt wird, wobei aber natürlich nicht aufser Acht zu lassen ist, dafs auch zahlreiche andere trockene Drogen eine solche Umwandlung von Gerbstoffen zu braunen oder gelben Stoffen erkennen lassen, ohne gerottet oder einem ähnlichen Prozesse unterworfen zu werden. Die Fabrikanten beurteilen den Kakaosamen nach der Farbe des Querschnittes und halten möglichst ausgesprochen braune Samen für die besten, welche also möglichst reich an Phlobaphen nnd möglichst arm an Gerbstoff sind, also wahrscheinlich den durch den Gerbstoff bedingten hẹrben Geschmack möglichst vollständig verloren haben.

Von einjgen Sorten lagen uns durch die gütige Vermittelung der Herren Wittekop \& Co. zugleich ungeröstete und geröstete 
Samen vor und es war damit die erwünschte Gelegenheit geboten, auch das Verhalten des Pigmentes beim Rösten zu studieren. Es hat sich nun herausgestellt, dal's beim Rösten die violette Farbe des Pigments in Braun von verschiedenen Nuancen übergeht und dal's der Farbstoff dabei die Fïhigkeit verliert, in bekannter Weise anf Alkalien zı reagieren. Da aber offenbar das Rösten bei den jetzigen vervollkommneten Finrichtungen sehr vorsichtig geschieht, so ist es meist leicht, weiter nach innen in den Samen noch Pigmentzellen mit unverändertem violettem Inhalt anfzufinden. Ebenso konnten wir in dem uns zu Gebote stehenden Kakaomassen und Pulvem immer mit Leichtigkeit noch unveränderten Farbstoff auffinden.

Auch diesmal ist den Stärkekörnern einige Aufmerksamkeit zugewendet worden. nachdem schon $188 \bar{i}$ anf die erheblichen Differenzen in der Grörse der Stürkekörner bei manchen Sorten hingewiesen war und es hat sich herausgestellt, dais die diesmal darauf untersuchten Sorten mit denen von 1887 gut ühereinstimmen (nur bei der Sorte von St. Thomas wurden 1887 die Körner bis 3 ", diesmal bis $5,3 \mu$ grol's gefunden). Wenn nun selbstverständlich ein abschliefsendes Urteil noch nicht möglich ist, so wollen wir doch dringend empfehlen, vorkommenden Falls darauf zu achten.

Wir lassen in dem Folgenden unsere bei der makrokopischen und mikroskopischen Untersuchung 23 verschiedener Handelssorten gemachten Beobachtungen, sowie die Reaktionen der Auszüge dieser Bohnen folgen, wodurch das in dem Obigen ausgeführte, wonach die zur Unterscheidung bislang angenommenen Merkmale unzureichend sind und daher keine Verwendung mehr finden können, Bestätigung findet.

\section{Allgemeine Kennzeichen und mikroskopischer}

\section{Befund.}

\section{Bahia.}

(Brasilien.)

Bohne: abgeplattet.

Schalen: zimmetfarbig.

Samenlappen: aufsen braun, Querschnitt rotviolett, bei der gerösteten Bohne braun.

Durchschnitts mafs: $22 \mathrm{~mm}$ Länge, $13 \mathrm{~mm}$ Breite $6 \mathrm{~mm}$ Dick $\theta$. 
Gewicht: 20 Samen wiegen $21 \mathrm{~g}$.

Mikrosk opischer Befund: deutlich differenzierte Pigmentzellen sind nicht zu erkennen. vielmehr nimmt das ganze Gewebe der Kotyledonen mit Natronlauge eine schwach grünliche Farbe an und wird mit Eisenchlorid dunkel. Der Inhalt des Parenchyms ist in den äufseren Parthien braun gefürbt, weiter nach innen farblos. Stärkekörner bis 7,2 . "l.

\section{Bahia.}

(Brasilien.)

Bohne: abgeplatitet.

Schale: rotbraun.

Samenlappen: aufsen schwarzbraun, Querschnitt dunkelviolett Durchschnittsma $\int \mathrm{s}$ : $25 \mathrm{~mm}$ Länge, $14 \mathrm{~mm}$ Breite, $7 \mathrm{~mm}$ Dicke. Gewicht: 20 Stück wiegen $27 \mathrm{~g}$.

Mikroskopisch er Befund: Pigmentzellen rotviolett, mit Eisenchlorid dunkel, mit Natronlauge zuerst ganz kurze Zeit blau, dann mit grüner Farbe sich lösend, nach einiger /eit gelb. Amylum bis $\bar{i}$ ".

Bohne: konvex.

\section{Para.}

(Brasilien.)

Schalen: rostfarbig. matt.

Samenlappen: aulsen schwarzbraun, Querschnitt braun bis graubraul.

Durchschnittsmal's: $22 \mathrm{~mm}$ Länge, $11 \mathrm{~mm}$ Breite, $5 \mathrm{~mm}$ Dicke.

Gewicht: 20 Stück wiegen $18 \mathrm{~g}$.

Mikroskopischor Befund: Pigmentzellen violett, mit Eisenchlorid meist rot, nur selten schwarz werdend, mit Natronlauge wie Bahia 2. Brauner Farbstoff des Parenchyms sehr spärlich.

Hohne: konvex.

\section{Antzko.}

Brasilien.

Schalen: zimmetfarbig.

Samenlappen: aufsen schwarzbraun, innen violettbraun, Querschnitt hellbraun, auch violett,

Durchschnittsmaf's: $20 \mathrm{~mm}$ Länge, $11 \mathrm{~mm}$ Breite, $6 \mathrm{~mm}$ Dicke.

Gewicht: 20 Stück wiegen $20 \mathrm{~g}$.

Mikrosk opischer B ef und: Pigmentzellen rotviolett mit einem Stich ins Braune, auffallend hlein. Mit Eisenchlorid werden sie schwarz, mit Natronlauge reagieren sie wie Bahia 2; aber nur schwach, offenbar ist also die Umwandlung des Gerbstofies in den violetten Farbstoff noch nicht weit vorgeschritten. Brauner Farbstoff im Parenchym fehlt fast ganz. Amylum 3,5 ". 
Die geröstete Bohne zeigt einen hellbraunen, auch grauen Querschnitt, die Pigmentzellen sind meist rotbraun, mit Eisenchlorid werden sie schwarz. in Natronlauge löst sich der Farbstoff farblos.

\section{Maracas. $\left.{ }^{*}\right)$}

(Brasilien.)

Boh ne: abgeplattet, auch konvex.

$\mathrm{Sch}$ alen: rotbraun.

Samenlappen: aufsen dunkelbraun, innen hellbraun, Querschnitt graubraun.

Durchschnittsm afs: $23 \mathrm{~mm}$ Länge, $13 \mathrm{~mm}$ Breite, $7 \mathrm{~mm}$ Dicke.

Gewicht; 20 Stück wiegen $27,5 \mathrm{~g}$.

Mikroskopischer Befund: Pigmentzellen mit violettem In. halt, der mit Eisenchlorid schwar\% wird und mit Natronlauge wie Bahia reagiert. Amylum 1,8-3,6 $\mu$.

Die geröstete Bohne ist im Querschnitt hellbraun, die Pigmentzellen sind gelbbraun, mit Eisenchlorid werden sie schwarz, mit Natronlauge werden sie einen Moment schwach rötlich und lösen sich dann farblos.

Bohne: konvex.

6. Canca.

(Columbien.)

Schalen: rostfarbig, auch graubraun.

Samenlappen: aufsen hellbraun, Querschnitt gelblich braun.

Durchschnitts ma fs : $23 \mathrm{~mm}$ Länge, $13 \mathrm{~mm}$ Breite, $10 \mathrm{~mm}$ Dicke.

Gewicht: 20 Samen wiegen $2 \bar{i}, \bar{j} \mathrm{~g}$.

Mikroskopischer Befund: Pigmentzellen mit violettem Inhalt fehlen, einzelne Zellen sind braun gefärbt, sie werden mit Eisenchlorid schwarz, wie auch das ganze Kotyledonengewebe meist etwas dunkler wird. Brauner Farbstoff des Parenchyms ist reichlich vorhanden. Amylum bis $\bar{i}, 2$, .

\section{Carracas.}

Venezuela.

Bohne: teilweise abgeplattet, teilweise konvex.

Schalen: ockerfarbig.

Samenlappen: aufsen dunkelbraun, innen hellbraun, oft mit violettem Ton.

Durchschnittsmafs: $23 \mathrm{~mm}$ Länge, $11,5 \mathrm{~mm}$ Breite, $9 \mathrm{~mm}$ Dicke.

Gewicht: 20 Samen wiegen 23,5 g.

Mikroskopischer Befund: Pigmentzellen mit dunkelviolettem Inhalt, mit Eisenchlorid tief dunkelrot, fast schwärzlich, mit Natron.

*) Ort in der Brasil. Provinz Bahia. Eine besonders geschätzte Ware; was gewöhnlich Bahia-Cacao genannt wird, ist nur mittelmälsige Ware. 
lauge wie Bahia 2, das Parenchym zeigt nur in den äul'seren Parthien etwas braunen Farbstoff.

\section{Garupano.}

(Venezuela.)

Bohne: abgepl attet.

Schalen: erdfarbiggrau.

Samenlappen: auísen dunkelbraun, innen rötlich. Querschnit violett.

Durchschnit smal's: $2+\mathrm{mm}$ Länge, $13,5 \mathrm{~mm}$ Breite, $6 \mathrm{~mm}$ Dicke.

Gewicht: 20 Stück wiegen $24 \mathrm{~g}$.

Mikroshopischer Befund: Pigmentzellen mit violettem Inhalt selten, dagegen das äulsere Gewebe violett gefärbt. Mit Eisenchlorid wird das ganze Gewebe dunkel, mit Natronlauge schwach grünlich. Das Parenchym mit reichlichem braunen Farbstoff: Amylum bis $13,5, \mu$. grols.

\section{Puerto Cabello.}

(Tenezuela.)

Bohne: konvex.

Schalen: ockertarbig, erdig.

Samenlappen: aufsen dunkelbraun, innen hellbraun.

Durchschnittsmafs: $23 \mathrm{~mm}$ Länge, $6 \mathrm{~mm}$ Breite, $13 \mathrm{~mm}$ Dicke. Gewicht: 20 Stück wiegen $26 \mathrm{~g}$.

Mikroskopischer B efund: Violetter Farbstoff fehlt ganz, einzelne Zellen zeigen schwach braungefärbten Inhalt, der sich mit Eisenchlorid wenig dunkler fürbt.

\section{Caraguez.}

(Ecuador.)

Bohne: abgeplattet und konvex.

Schalen: rostfarbig, of mehr grau.

Samenlappen: aulen dunkelbraun.

Durch schnitts mal's: $23 \mathrm{~mm}$ Länge, $13 \mathrm{~mm}$ Breite, $7,3 \mathrm{~mm}$ Dicke.

Gewicht: 20 Samen wiegen $26 \mathrm{~g}$.

Mikroskopischer Beflund: Die Sorte zeigt nicht durchweg gleiche Beschaffenheit des Samens, es fanden sich solche, die 1) einen braunen Querschnitt hatten und rötliche Pigmentzellen zeigten, sowie reichlich braunen Farbstoff im Parenchym und 2) solche, die einen violetten Querschnitt hatten und violette Pigmentzellen, aber keinen braunen Farbstoff im Parenchym. Bei beiden Sorten wurde das Pig. ment mit Eisenchlorid dunkel und reagierte mit Natronlauge wio Bahia ?.

Amylum 3,6-5,2. $\mu$. 


\section{Machala-Ginayaquil.}

Bohne: konvex.

(Ecuador.)

Schalen: zimmtfarbig.

Samenlappen: aufsen dunkelbraun, innen hellbraun.

Durchschnitts mafs: $24 \mathrm{~mm}$ Länge, $13 \mathrm{~mm}$ Breite, $7 \mathrm{~mm}$ Dicke. Gewicht: 20 Stück wiegen $33 \mathrm{~g}$.

Mikroskopischer Bef und: Pigmentzellen mit dunkelviolettem, weiter nach innen hellerem, fast blauen Inhalt; mit Eisenchlorid rot werdend, nach einiger Zeit die meisten Zellen schwarz; mit Natronlauge wie Bahia 2.

Die geröstete Bohne zeigt einen graubraunen Querschnitt, das Pigment ist in den äufseren Partien in braun umgewandelt, in den inneren unverändert. In Natronlauge löst es sich farblos.

\section{Arriba Guayaquil.}

\section{(Ecuador.)}

Sommer-Ernte.

Bohne: abgeplattet, auch konvex.

Schalen: rostfarbig.

Samenlappen: braun.

Durchschnit tsmal's : $24 \mathrm{~mm}$ Länge. $15 \mathrm{~mm}$ Breite, $7,5 \mathrm{~mm}$ Dicke.

Gewicht: 20 Stück wogen $26,5 \mathrm{~g}$.

Mikroskopischer Befund: Die Samen sind nicht von gleichmälsiger Beschaffenheit, es kommen vor: 1) solche mit dunkelbraunviolettem Querschnitt und ohne violette Pigmentzellen: dagegen sind einige Zellen schwach braun, sie färben sich mit Eisenchlorid dunkel und geben mit Natronlauge keine Reaktion.

2) solche mit grauem Querschnitt, die braunen Farbstoff im Parenchym und l'igmentzellen mit violettem Inhalt zeigen, welche letzterer mit Eisenchlorid erst rot, dann schwarz wird und mit Natronlauge wie Bahia reagiert. Amylum bis $\tau \mu$.

Geröstete Bohnen derselben Sorte zeigen einen braunen Querschnitt und rotbraune Pigmentzellen, die sich mit Eisenchlorid schwärzen und mit Natronlauge keine Farbenreaktion geben.

\section{Arriba Guayaquil.}

B ohne: abgeplattet.

$$
\text { Winter-Ernte. }
$$

Schalen: rostfarbig.

Samenlappen: aul'sen braun, Querschnitt rötlich braun.

Durchschnitts ma fs: $25 \mathrm{~mm}$ Länge, $14 \mathrm{~mm}$ Breite, $7 \mathrm{~mm}$ Dicke. Gewicht: 20 Stück wiegen $36 \mathrm{~g}$. 
Mikroskopischer Befund: Pigmentzellen mit violettem Inhalt selten, mit Eisenchlorid schwarz, mit Natronlauge wie Bahia 2. Brauner Farbstofi im Parenchym reichlich.

\section{Guayaquil.}

(Balao.)

Bohne; konvex.

Schalen: rostfarbig.

Samenlappen: aulsen dunkelbraun, innen hellbraun.

Durchschnittsmals: $23 \mathrm{~mm}$ Länge, $13 \mathrm{~mm}$ Breite, $8 \mathrm{~mm}$ Dicke.

(iewicht: 20 Stück wiegen $28,7 \mathrm{~g}$.

Mikroskopischer Befund: Die Samen sind ungleichmäl'sig, man unterscheidet folgende: 1) solche mit braunem Querschnitt und braunvioletten Pigmentzellen, die mit Eisenchlorid dunkel werden und mit Natronlauge wie Bahia 2 reagieren und 2) solche mit violettem Querschnitt, die nur spärliche, ganz schwach violett gefärbte Pigmentzellen zeigen, die mit Eisenchlorid schwarz werden. Mit Natronlauge nimmt das ganze Gewebe der Samenlappen einen grünlichen Ton an. Beide Arten zeigen im Parenchym eine geringe Menge braunen Farbstoff. Amylum 3,5-5,2,, .

\section{Domingo.}

(Westindien.)

Bohne: abgeplattet.

Schalen: schrnutzig-grau.

Samenlappen: aufsen graubraun. Querschnitt gelblich-braun.

Durchschnittsmafs: $22 \mathrm{~mm}$ Länge, $13 \mathrm{~mm}$ Breite, $6 \mathrm{~mm}$ Dick $\theta$. Gewicht: 20 Samen wiegen $20,2 \mathrm{~g}$.

Mikroskopis cher Befund: Pigmentzellen rotviolett, mit Eisen. chlorid schwarz mit Natronlauge wie Bahia 2, brauner Farbstoft des Parenchyms spärlich.

\section{Dominica.}

(Westindien.)

Bohne: abgeplattet.

Schalen: rostfarbig.

Samenlappen: schwarzbraun. Durchschnitt grauviolett.

Durchschnittsmafs: $25 \mathrm{~mm}$ Länge, $13 \mathrm{~mm}$ Breite, $7 \mathrm{~mm}$ Dicke. Gewicht: 20 Stück wiegen $23,2 \mathrm{~g}$.

Mikroskopischer Befund: Pigmentzellen rotviolett, mit Eisenchlorid einige rot, die meisten schwarz, mit Natronlauge wie Bahia 2. Das Parenchym besonders in den äulseren Parthien mit braunem Farbstoff. Amylum bis 5,4 " grofs. 


\section{Granada.}

(Westindien.)

Bohne: teilweise abgeplattet, teilweise konvex.

Schalen: zimmtfarbig.

Samenlappen: aufsen schwarzbraun, Querschnitt gelbbraun bis rotviolett

Durchschnittyma l's : $23 \mathrm{~mm}$ Länge, $13 \mathrm{~mm}$ Breite, $8 \mathrm{~mm}$ Dicke.

Gewicht: 20 Stück wiegen $19,5 \mathrm{~g}$.

Mikroskopischer Befund: Pigmentzellen violett, mit Eisenchlorid schwarz, zugleich auch das ganze Gewebe etwas dunkler, mit Natronlauge wie Bahia 2.

Amylumkörner bis $\bar{\tau}$ " grofs. Auf́ser den Amylumkornern fanden sich bei dieser Sorte Gerbstoffkörner von der Gröfse der Amylumkörner.

Bohne: abgeplattet.

18. st. Lucia.

(Westindien.)

Schale: graubraun.

Sam enlappen: aul'sen dunkelbraun. innen hellbraun bis graubraun.

Durch sch nitt s m a Is: $2.2 \mathrm{~mm}$ Länge, $12,5 \mathrm{~mm}$ Breite, $5,5 \mathrm{~mm}$ Dicke

Gewicht: 20 Stück wiegen $23 \mathrm{~g}$.

Mikroskopischer Befund: Violette Pigmentzellen selu selten. mit Eisenchlorid mehr rot, mit Natronlange wie Bahia 2. Braulter Farbstoff in den Samenlappen reichlich.

\section{Trinidad.}

Bohne: abgeplattet.

(Westindien.)

Schalen: rostfarbig.

Samenlappen: aufsen dunkelbraun, Querschnitt rotviolett.

Durchschnitts mal's: $23 \mathrm{~mm}$ Länge, $13 \mathrm{~mm}$ Breite, $7 \mathrm{~mm}$ Dicke.

(jewicht: 20) Stück wiegen $19,5 \mathrm{~g}$.

Mikrosk opischer Befund: Pigmentzellen fehlen. Brauner Farbstoff im Parenchym in den äulseren Parthien.

Bohne: honvex.

\section{1). ceylon.}

Schalen: rötlichbraun.

Samenlappen: hellbraun. Querschnitt braun.

Durchschnitts ma s: $21 \mathrm{~mm}$ Länge, $13 \mathrm{~mm}$ Breite, $8 \mathrm{~mm}$ Dicke.

Gewicht: 20 Stiicke wiegen $17,3 \mathrm{~g}$.

Mikroskopischer Befund: Pigmentzellen fehlen. Mit Eisenchlorid wird das ganze Gewebe der Samenlappen otwas dunkler, einige Zellen schwar\%. Brauner Farbstofi der samenlappen deutlich. Amvlum bis $T$, 
Bohne: konvex.

\section{Jara.}

Schalen: kuptertarbig.

Samenlappen: aufsen braun, Querschnitt braun.

Durchschnittsmafs: $22 \mathrm{~mm}$ Länge, $13 \mathrm{~mm}$ Breite, $8 \mathrm{~mm}$ Dicke.

Gewicht: 20 Stück wogen $18,5 \mathrm{~g}$.

Mikroskopischer Befund: Pigmentzellen spärlich vorhanden, mit sehr hellviolettem, zuweilen hellbraunem Inhalt, der mit Eisenchlorid dunkel wird. Das Parenchym mit mäfsigem braunen Farbstoff,

Bohne: abgeplattet.

\section{Kamerun.}

Schalen: dunkel zimmetfarbig.

Samenlappen: aulsen dunkelbraun, innen violettbraun.

Durchschnitts ma fs: $24 \mathrm{~mm}$ Länge, $13,5 \mathrm{~mm}$ Breite, $6 \mathrm{~mm}$ Dicke.

Gewicht: 20 Stück wiegen $24 \mathrm{~g}$.

Mikroskopischer Befund: Pigmentzellen rotviolett, weiter nach innen hellblau, mit Eisenchlorid dunkel, mit Natronlauge wie Bahia ?.

Bohne: abgeplattet.

\section{3. st. Thome.}

\section{(Afrika.)}

Schalen; graubräunlich.

Samenlappen: aufsen schwarzlraun, Querschnitt grauviolett, auch entschieden violett.

Durchschnittsma $\mathrm{s}$ : $25 \mathrm{~mm}$ Lünge, $13 \mathrm{~mm}$ Breite, $7 \mathrm{~mm}$ Dicke.

Gewicht: 20 Stück wiegen $22 \mathrm{~g}$.

Mikroskopis cher Bef und: Pigmentzellendunkelviolett(zuweilen fast ganz fehlend), mit Eisenchlorid rot, mit Natronlauge wie Bahia 2. Amylum 5,3 $\mu$. Brauner Farbstoff' im Parenchym spärlich.

2. Reaktionen der Auszüge von Bohnen verschiedener Provenienz.

Nach Vorschrift Trojanowsky's wurden 2 is Kotyledonenpulver mit $2 \mathrm{~g}$ Zuckerpulver fein verrieben, mit $30 \mathrm{~g}$ Wasser ubergossen, 24 Stunden stehen gelassen und filtriert. Je $1 \mathrm{ccm}$ des Filtrats wurde mit den nachbezeichneten Reagentien versetzt und die eintretenden Reaktionen beobachtet. Dieselben sind in der folgendon Tabelle zusammengestellt, obwohl sie eine Bedentung für die Unterscheidung von Kakaostandardmustern nicht haben, weil ess nur durch Anführung derselben möglich ist, iluen zweifelhaften Wert darznthun. 
PAGES MISSING FROM 601 TO 608 